\title{
An e-Learning Application Based on the Semantic Web Technology
}

\author{
Boyka Gradinaroval, Ognjan Zhelezov ${ }^{2}$ and Milena Gradinarova ${ }^{3}$ \\ 1 Technical University of Varna, 9000 Varna, Studrntska,Sre. 1,e-mail: \\ inflot@mbox.digsys.bg \\ 2 Technical University of Varna, 9000 Varna, Studrntska,Sre.1,e-mail: \\ ognyanz@yahoo.com \\ 3 Sofia University, 15, Car Osvoboditel bul.,1504 Sofia,mimeto@eol.com
}

\begin{abstract}
The paper describes a framework for the implementation of an elearning system based on the Semantic Web, using software agents and Java Web Services. Hopefully we have elucidated the enormous potential of making web content machine-understandable. One of the killer applications for the Semantic Web might prove to be related to e-learning, considering the amount of research in this sector and the advantages those applications bring to the table compared to existing web-based learning courses.
\end{abstract}

\section{Introduction}

Increasingly, The World Wide Web (WWW) is used to support and facilitate the delivery of teaching and learning materials. This use has progressed from the augmentation of conventional courses through web-based training and distance learning to a newer form of WWW-based education, e-learning [11]. E-learning is not just concerned with providing easy access to learning resources anytime, anywhere, via a repository of learning resources, but is also concerned with supporting such features as personal definition of learning goals, synchronous and asynchronous communication, and collaboration between learners and between learners and instructors [2] [13].

Researchers have proposed that in an e-learning environment the educational content should be oriented around small modules (or learning objects) coupled with associated semantics (or metadata) to be able to find what one wants, and that these modules be related by a "dependency network" or "conceptual web" to allow individual instruction. Such a dependency network allows, for example, the learning objects to be presented to the student in an orderly manner, with prerequisite material being presented first. Additionally, in an e-learning environment students must be

Please use the following format when citing this chapter:

Gradinarova, B., Zhelezov, O., Gradinarova, M, 2006, in International Federation for Information Processing, Volume 210, Education for the $21^{\text {st }}$ Century-Impact of ICT and Digital Resources, eds. D. Kumar, and Tumer J., (Boston: Springer), pp. 75-82. 
able to add extra material and links (i.e., annotate) to the learning objects for their own benefit or for that of later students [10] [15] [17].

This framework lends itself to an implementation based on the Semantic Web, incorporating cooperating software agents, which additionally make use of appropriate web services to provide the functionality. We are developing an elearning application using these technologies. The facilities that the application will provide include allowing the e-learning content to be created, annotated shared and discussed together with supplying such lecture notes, student portfolios, group projects, information pages, discussion forums and question-and-answer bulletin boards. The application will allow students to benefit from more interaction with their peers (for example, sharing the resources found on the web), as well as with the instructors. Students will see more ideas, more depth to those ideas, and a faster turnaround time in getting them. They will be able to learn from each other's notes, the questions that are asked and the answers given. It will provide an easy way for students to share and archive information, whether of general interest or specific to a group project they are involved in.

This paper describes the important and strategic role that the Semantic Web will play in this development - how Semantic Web concepts can support facets of elearning and describes an e-learning application framework based on these concepts.

\section{Semantic Web}

The current WWW is a powerful tool for research and education, but its utility is hampered by the inability of the user to navigate easily the nefarious sources for the information he requires. The Semantic $W e b$ is a vision to solve this problem. It is proposed that a new WWW architecture will support not only web content, but also associated formal semantics [4]. The idea is that the web content and accompanying semantics (or metadata) will be accessed by web agents, allowing these agents to reason about the content and produce intelligent answers to users' queries.

The Semantic Web, in practice, comprises a layered framework: an XML layer for expressing the web content; a Resource Description Framework (RDF) [8] layer for representing the semantics of the content; an ontology layer for describing the vocabulary of the domain; and a logic layer to enable intelligent reasoning with meaningful data [18].

XML was designed as a simple, flexible way of transporting structured documents across the web. With XML, "tags", or hidden labels may be created such as <address> or <title> - that annotate web pages or sections of text within a page. XML is machine-readable, i.e., programs can read and understand it, but the program developer has to know what the page writer uses each tag for. In other words, XML allows users to add arbitrary structure to their documents but says nothing about what the structures mean [5].

The meaning of the document content is expressed with RDF that is simply a data model and format that allows the creation of machine-readable data. It comprises a set of triples, i.e., three Universal Resource Identifiers (URIs) that may be used to describe any possible relationship existing between the data - subject, 
object and predicate [7] [16]. Thus, all data stored in the system is easily readable and processible. It is important to note that RDF provides the syntax, but not the actual meaning of the properties we ascribe to the data. For example, it does not define what data properties such as Title or Category or Related-To mean. Properties like these are not standalone; they come in packages called domain vocabularies. A learning object, for example, may include a set of properties such as Course, Sub-Section, Author, Title, Similar-To, Difficulty-Level, and Rating. Thus, for every domain there is a need for a specific ontology to describe the vocabularies and to make sure they are compatible.

Ontologies in the context of the Semantic Web are specifications of the conceptualization and corresponding vocabulary used to describe a domain [12]. Any semantic on the web is based on an explicitly-specified ontology, so different Semantic Web applications can communicate by exchanging their ontologies. Several representation schemes have been defined for the ontology layer. The most popular one, the Ontology Interchange Language (OIL) combined with the DARPA Agent Markup Language (DAML), DAML+OIL, provides a rich set of language structures with which to create ontologies and to markup information so that it is machine understandable.

The logic layer part of the Semantic Web is not fully developed yet. Its implementation will allow the user to state any logical principles and permit the computer to infer new knowledge by applying these principles to the existing data. Since there are many different inference systems on the web that are not completely interoperable, the vision is to develop a universal logic language for representing proofs - systems will then be able to export these proofs into the Semantic Web [1].

Within an e-learning framework, the Semantic Web provides the technology that allows a learning object to be (i) described with metatdata, and this description to be extended indefinitely (by anyone, not just the creator); (ii) annotated with personal notes and links by anyone; (iii) extended in terms of content, allowing multiple versions to exist; (iv) shared by, and communicated to, anyone who has expressed an interest in such content; and (v) certified, for example, as a quality learning resource; and more [16]. The e-learning application described in [16] embraces this functionality.

\section{Application}

The e-learning application framework is organized around a learning object repository that exclusively contains metadata [10]. In fact, it is a repository of the URIs of learning objects, such as Web sites, word documents, PDF files, PowerPoint presentations, etc., plus descriptions about these objects. No actual objects are stored in the repository, only links (i.e., UPLs). A most important aspect of the application is the metadata stored regarding each and every URL. The addition of such semantic information to the URLs will be done via RDF, using dynamically-generated Web pages, personalized for every user. Since the information is stored in the form of machine-understandable RDF statements, it can be used by the application agents. In order to give meaning to these RDF statements, we are developing our own e- 
learning ontology (vocabulary) to be used within our application. It is designed using DAML (DARPA Agent Markup Language) and will ensure compatibility of the metadata attached by various users.

In this section we describe some of the main features of our e-learning application that are enabled with Semantic Web technologies.

A main concept of our application is that it enables dynamic course creation and extension/modification, i.e., the contents of the course are extremely flexible. Most of the content will initially be provided by the instructor in a form of links to small learning objects. All of these links will be annotated with RDF statements that will provide a description about the document/URL linked. Researchers have proposed three forms of annotation: concept annotation, context annotation and structure annotation [Stojanovic]. For the e-learning domain, annotations might include the context in which the document is placed, links toward other similar objects, the relationship to other documents (some learning objects might be prerequisites for access to others), rating (which will be updated with other users' ratings), etc. Importantly, however, the student will also be able to add his own, further annotations to personalize and enrich the learning material. Moreover, these annotations are not necessarily collected in one document - they may be dispersed throughout the web.

An important feature of the application is the use of e-protocols for assessing student performance, a feature that, additionally, significantly enriches the learning potential of the system. Since a portfolio simply represents an organized collection of completed work, the items in the portfolio can be thought of themselves as learning resources. Hence, the e-portfolio collection provides yet another repository of learning objects that may be used later by other students. For this, the e-portfolios will need to contain RDF descriptions of their metadata. So, when submitting a document to his portfolio, the student will be required to annotate the document through a template-driven Web page. Using Semantic Web, e-portfolios become content management systems, allowing not only the publishing of documents, but also the dissemination of metadata about these documents and the structure of courses, as well subjective annotations of e-resources [16].

If there is an abundance of learning objects available for the students, there needs to be a way for them to distinguish quality ones. Again, annotation of the learning objects supports certification/rating - instructors are able to certify a specific content as being a quality learning resource.

An intriguing future extension being investigated is the inclusion of contextaware links. Imagine a student who has opened some learning object to study. If it is advanced material, the student might have problems comprehending some of the terms. The browser, after querying metadata servers about such terms, may automatically place links for the more difficult terms/concepts in the learning object, connecting them with other learning objects that might make the student's understanding easier. Even though such browsers exist today, the application of this technology using the Semantic Web is still in its nascent stage. One of the first steps toward context-aware browsing has been the introduction of a concept browser, Conzilla, which creates an overview of the different concepts under study by supporting a separation of their context and their content [9]. 


\section{Implementation}

There are two types of agents used in the application. Student/Agent and Instructor/Agent, both of them implemented as Java classes. Users are served by the appropriate agents, which parse the metadata and tailor the user interface to satisfy the user's needs, whether student or instructor. For example, if a student encounters difficulties with a section of a course, he may notify the Student/Agent of this fact, and whenever a new resource related to a section appears on the web, the agent will insert a link to this resource on the student's personal page. The agents interact and communicate between each other by means of Java Web Services using the Simple Object Access Protocol (SOAP). The general structure is illustrated in Figure 1.

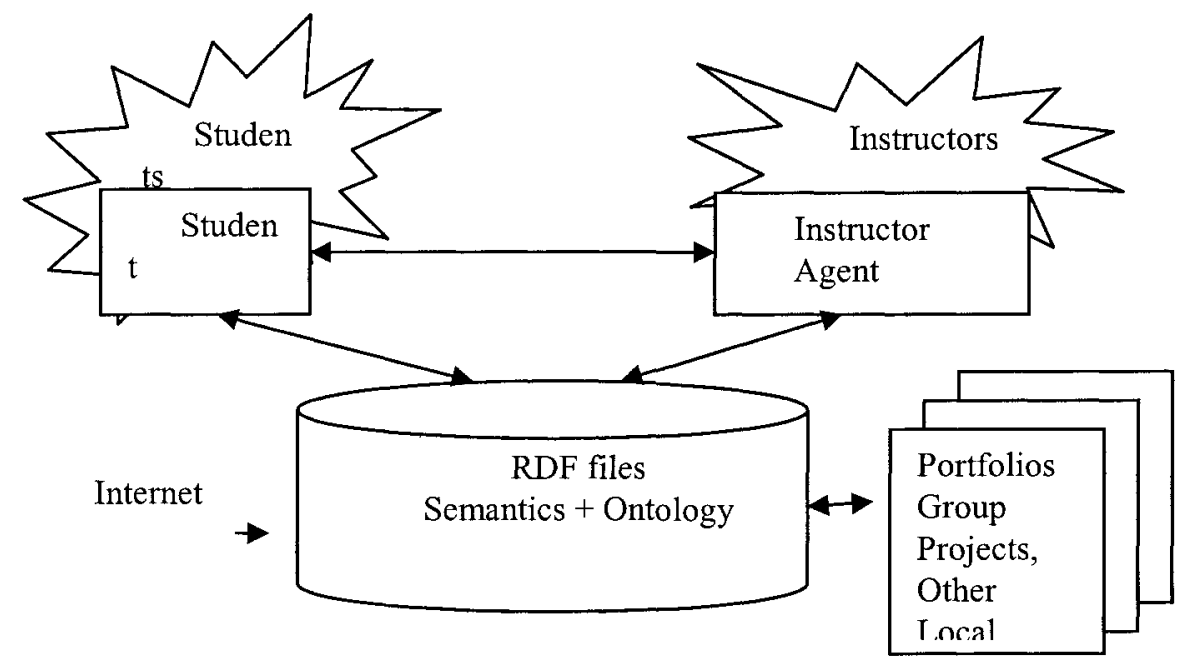

Figure 1. The general structure of the system

Users will add any metadata (whether it be personal annotation, certification, etc.) to a document referenced via the RDF learning object repository through dynamic (Java Server) web pages. For the end user, this process of annotation is identical the action of filling out fields in a web form. After the user submits the form, the application automatically converts this additional information to a set of RDF statements using the Jena API (see below) and then adds them to the existing RDF statements for this document in the repository. Because the RDF specifications provide XML syntax for writing down and exchanging RDF statements (called $\mathrm{RDF} / \mathrm{XML}$ ). The repository is implemented as a set of RDF/XML files. However, the $\mathrm{RDF} / \mathrm{XML}$ syntax is quite complex, and developing an RDF parser is not a trivial task.

Motivated by the need for an RDF parser, we are using a Semantic Web toolkit called Jena for developing our application. Jena is a Java API for manipulating RDF 
statements, capable of parsing RDF statements and storing them as graphs (or models), thereby allowing straightforward manipulation of the RDF [14]. Additionally, Jena provides support for converting DAML-specified ontologies to an RDF form, and also implements a Resource Description Query Language (RDQL), an SQL-like query language for RDF statements.

To illustrate the Jena RDF API, consider the following simple example of an RDF statement describing a learning object. The main component of the statement is represented with the Resource class in Jena, and is always a URL. For instance, let $\mathrm{http}: / /$ some.site/DiffGeometry be the URL for an e-book about differential geometry. This resource will be represented as a node in the graph model, whereas the properties it might possess are marked as edges. One of the properties for the e-book resource may be "Title", and the name of the property is also a URL. The property has a value, "Elementary Differential Geometry", in this case and this value is again marked by a node in the graph. Another property may be "Similar To", which points to another resource (URL) holding similar information as http://.../AnotherGeomethryBook. The following is the Java source-code required to create this RDF model in Jena:

Static String objectURL = "http://some.site//DiffGeometry";

Static String similarToURL= "http://..// AnotherGeomethryBook";

//create the empty model

Model model = new ModelMem();

//create the resource

Resource learningObject $=$ model.createResource $($ objectURL $)$;

$/ /$ add first property (title)

learningObject.addProperty(propertyURL, "Introduction to Differential

Geometry");

//add the second property (similar to)

//the property does not have a literal value, it is a resource

Resource secondLearningObject $=$ model.createResource $($ similarToURL $)$;

LearningObject.addProperty(anotherPropertyURL, secondLearningObject);

A list of the technologies used in the implementation of the e-learning application includes Java 2 Platform, Enterprise Edition v1.3 (J2EE), Java Web Services Developer Packv1.1 (Java WSDP), JBuilder v6.0, Jakarta-Tomcat Server v3.2.3, Microsoft SQL Server 2000, and Jena Semantic Web Toolkit v1.6.1.

\section{Related work}

Stojanovic et al [18] describe an e-learning scenario based on the Semantic Web, in particular concentrating on ontologies for e-learning objects. This group is 
associated with the Learning Lab Lower Saxony, which itself is a partner in the Wallenberg Learning Network.

Naeve et al [16] describe an e-learning framework, again based on the Semantic Web, that discusses Semantic Web technologies and peer-to-peer services for the search, consortium comprising Swedish and German universities developing a P2P network for the exchange of educational resources.

\section{Conclusions and future work}

We have described a framework for an e-learning application based on the Semantic Web technology, incorporating cooperating software agents that additionally make use of appropriate web services to provide the functionality. With our paper, hopefully we have elucidated the enormous potential of making web content machine-understandable. Just as in the case of the present web, the potential of a globally-linked Semantic Web network will slowly become realized as the number of active users increases. One of the killer applications for the Semantic Web might prove to be related to e-learning, considering the amount of research in this sector and the advantages those applications bring to the table compared to existing webbased learning courses.

We are currently developing the application. Additional features will be included in the application as we gain experience with this new technology. This is a relatively young field with the promise for enormous growth.

\section{References}

1. Alschuler,L.Going to Extremes 2000 http://www.hml.com/lpt/2000/09/13/exstremes.html.

2. Andrews, K, A. Nedoumov, N. Scherbakov. Embeding Courswere into Internet: Problems and Solutions. ED-Media Conference, 1995.

3. Barker, P. Developing Teachig Webs: Advantages, Problems and Pitfalls. Educational Multimedia, Hypermedia \& Telecommunication (AACE) Conference, 2000.

4. Bemers-Lee, T. Semantic Web Roadmap http://www.w3.org/Designlssues/Sementic.html.

5. Bemers-Lee, T., J. Hendier, O. Lassila. The Semantic Web. Scintific American May 2001.

6. Bemers-Lee, T. Semantic Web on HML. HML 2000 Conference 2000.

7. Bray, T. RDF and Metadata. 1998 http:// www.hml.com/pub/a/98/06/rdf.html. 
8. Bray, T. is What is RDF? $2001 \mathrm{http}: / / \mathrm{www} . \mathrm{hm} l . c o m / p u b / a / 2001 / 01 / 24 / \mathrm{rdf}$.html.

9. Conzilla Concept Browser. Available from http://conzilla.org.

10. Downes, S. Learning Objects: Resources For Distance Education Worlwide. International Review of Research in Open and Distance Learning. 2001.

11. Drucker, P. Need to Know - Integrating e- Learning with High Velocity Value Chains. Delphi Group White Paper. 2000 www.delphigroup.com.

12. Gruber, T. A translation approach to portable ontology specification. Knowledge Acquisition, vol.5 1993.

13. Helic, D, H. Maurer, N. Scherbakov. Web-based Training: What We Expect from the System. ICCE, 2000.

14. Jena. Available from http://www.hpl.hp.com/semweb/jena.htm.

15. Maurer, H, M. Sapper. E-Learning Has to be Seen as Part of General Knowledge Management. ED-MEDIA Conference, 2001.

16. Naeve, A. M. Nilsson, M. Palmer. E-learning in the Semantic Age. The $2^{\text {nd }}$ European Web-based learning, Environments Conference, 2001.

17. Naeve, A, M. Nilsson, M. Palmer. The Conceptual Web - Our Research Vision. The $1^{\text {st }}$ Semantic Web Working Symposium, 2001.

18. Stojanovic L. S Staab, R Studer. E-learning based on the Semantic Web. WebNet2001 World Conference on the WWW and Internet, 2001 